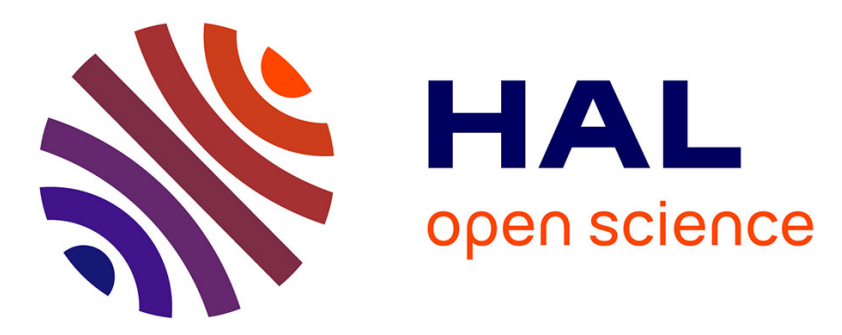

\title{
Different composite voxel methods for the numerical homogenization of heterogeneous inelastic materials with FFT-based techniques
}

\author{
Charles Mareau, Camille Robert
}

\section{- To cite this version:}

Charles Mareau, Camille Robert. Different composite voxel methods for the numerical homogenization of heterogeneous inelastic materials with FFT-based techniques. Mechanics of Materials, 2017, 105, pp.157-165. 10.1016/j.mechmat.2016.12.002 . hal-01433013

\section{HAL Id: hal-01433013 https://hal.science/hal-01433013}

Submitted on 12 Jan 2017

HAL is a multi-disciplinary open access archive for the deposit and dissemination of scientific research documents, whether they are published or not. The documents may come from teaching and research institutions in France or abroad, or from public or private research centers.
L'archive ouverte pluridisciplinaire HAL, est destinée au dépôt et à la diffusion de documents scientifiques de niveau recherche, publiés ou non, émanant des établissements d'enseignement et de recherche français ou étrangers, des laboratoires publics ou privés. 


\title{
Different composite voxel methods for the numerical homogenization of heterogeneous inelastic materials with FFT-based techniques
}

\author{
Charles Mareau*, Camille Robert \\ Arts et Métiers ParisTech, Campus d'Angers, LAMPA, 2 bd du Ronceray, 49035 Angers Cedex 1, France
}

Keywords:

Homogenization

Interfaces

FFT method

Heterogeneous materials

\begin{abstract}
A B S T R A C T
FFT-based homogenization methods aim at calculating the effective behavior of heterogeneous materials with periodic microstructures. These methods operate on a regular grid of voxels, and hence require an appropriate spatial discretization of periodic microstructures. However, when different microstructural length scales are involved, it is not always possible to have sufficient spatial resolutions to explicitly consider the influence of fine microstructural features (e.g. voids, second-phase particles). To circumvent this difficulty, one solution consists of using composite voxel methods to define the effective properties and the effective internal variables of heterogeneous voxels.

In this work, different composite voxel methods are proposed to deal with inelastic materials with multiple length scales. These methods use simple homogenization rules to calculate the effective behavior of heterogeneous voxels. The first part of this paper is dedicated to the description of the composite voxel methods, which are based either on the Voigt, laminate structure or Mori-Tanaka approximations. In the second part, these methods are used to model the elasto-plastic behavior of a pearlitic steel polycrystalline aggregate. According to the results, the Voigt approximation, which ignores morphological features, is not appropriate for treating heterogeneous voxels. When morphological information is accounted for, with either the laminate structure or Mori-Tanaka approximations, a better agreement with experimental observations is obtained. Though none of these methods is universal, they offer some possibilities to investigate the mechanical behavior of heterogeneous materials involving multiple length scales.
\end{abstract}

\section{Introduction}

FFT-based homogenization methods, originally proposed by Moulinec and Suquet (1998) and Müller (1996), provide a convenient way of estimating the effective properties of heterogeneous materials with periodic microstructures. For instance, in a mechanical context, FFT-based methods have been used to investigate the behavior of different types of heterogeneous solids, including hyperelastic (Lahellec et al., 2003; Kabel et al., 2014), thermoelastic (Vinogradov and Milton, 2008; Anglin et al., 2014), plastic (Michel et al., 2001), viscoplastic (Lebensohn, 2001), viscoelastic (Figliuzzi et al., 2016) and elasto-viscoplastic (Lebensohn et al., 2012; Suquet et al., 2012; Eisenlohr et al., 2013; Mareau et al., 2013; Robert and Mareau, 2015; Mareau and Daymond, 2016) materials. Some recent applications have also used FFT-based methods to deal with nonlocal plasticity (Lebensohn and Needleman, 2016) and dislocation mechanics (Berbenni et al., 2014; Brenner et al., 2014).
Independently on constitutive relations, FFT-based homogenization methods aim at finding the solution to the integral equations deriving from the application of compatibility and static equilibrium conditions. For the numerical resolution of the corresponding equations, different iterative procedures have been proposed (Moulinec and Suquet, 1998; Eyre and Milton, 1999; Michel et al., 2001; Monchiet and Bonnet, 2012). As shown in the comparative study of Moulinec and Silva (2014), the efficiency of these procedures depends on the property contrast. Also, instead of using a fixed-point algorithm, an alternative strategy, which is based on the conjugate gradient method, has been proposed by Zeman et al. (2010) to improve convergence properties. This idea has been extended to non-linear constitutive relations by Gélébart and Mondon-Cancel (2013).

Whatever the iterative procedure is, and except for some specific cases, FFT-based methods do not provide exact solutions to the integral equations, but only numerical approximations whose accuracy depend on different factors.

First, the numerical resolution of integral equations requires the introduction of a homogeneous reference medium to which a Green operator is associated. As shown in the work of 
Brisard and Dormieux, 2012; Willot, 2015; Willot and Pellegrini, 2008, alternative definitions of the Green operator may contribute to improving both the accuracy of the stress and strain fields and the convergence properties.

Second, FFT-based homogenization methods operate on a regular grid of voxels, and hence require an appropriate spatial discretization of periodic microstructures. Evidently, the accuracy of the numerical approximation is largely influenced by the spatial resolution used for discretizing the volume element.

Third, within the volume of a voxel, different constituents may exist. This situation is for instance encountered in interfacial voxels which connect multiple domains with different properties. For the specific case of interfacial voxels, with either a linear (Gélébart and Ouaki, 2015; Kabel et al., 2015) or a non-linear behavior (Kabel et al., 2016a; 2016b), the solution quality can be improved by using some simple homogenization rules to define the effective behavior of such voxels.

When multiple length scales are involved in a microstructure, different constituents may also exist within a voxel. Indeed, when fine microstructural details exist (e.g. nano-particles, micro-voids), high spatial resolutions are usually needed to explicitly consider such details. The computational cost is however often prohibitive and some alternative strategies have to be developed. In this work, different composite voxel methods are proposed to circumvent this difficulty. These methods treat voxels as heterogeneous media and use homogenization techniques to compute the effective behavior.

This paper is organized as follows. In the first part, the equations governing the mechanical behavior of heterogeneous materials with periodic microstructures are given and the strategy for numerically solving these equations using FFT techniques is recalled. Then, different composite methods for treating heterogeneous voxels are proposed for materials exhibiting an inelastic behavior. Finally, to demonstrate the interest of such methods, the elastoplastic behavior of a pearlitic steel polycrystalline aggregate is investigated. The different homogenization methods are employed to consider the characteristic two-phased fine microstructure of pearlite. The relevance of the proposed composite voxel methods is discussed from numerical results.

\section{Field equations}

To represent a heterogeneous material with periodic microstructure, a unit cell with volume $V$ and boundary $\partial V$ is considered. For the specific case of periodic boundary conditions, the displacement vector $\boldsymbol{u}(\boldsymbol{x}, t)$, which is associated with a material point occupying a position $\boldsymbol{x}$ at time $t$, is decomposed as follows:

$\boldsymbol{u}(\boldsymbol{x}, t)=\tilde{\boldsymbol{u}}(\boldsymbol{x}, t)+(\boldsymbol{E}(t)+\boldsymbol{\Omega}(t)) \cdot \boldsymbol{x}$

where $\tilde{\boldsymbol{u}}(\boldsymbol{x}, t)$ is the periodic part of the displacement field, $\boldsymbol{E}(t)$ and $\boldsymbol{\Omega}(t)$ are respectively the macroscopic strain and rotation tensors. The effective response of the heterogeneous material is given by the evolution of the macroscopic stress tensor $\boldsymbol{\Sigma}(t)$, which depends on microstructural features, local constitutive relations and prescribed loading conditions. The macroscopic quantities $\boldsymbol{\Sigma}(t), \boldsymbol{E}(t)$ and $\boldsymbol{\Omega}(t)$ are connected to their microscopic counterparts $\boldsymbol{\sigma}(\boldsymbol{x}, t)$, $\boldsymbol{\epsilon}(\boldsymbol{x}, t)$ and $\boldsymbol{\omega}(\boldsymbol{x}, t)$ with the classical averaging relations of homogenization theory:

$$
\begin{aligned}
& \boldsymbol{\Sigma}(t)=\frac{1}{V} \int_{V} \boldsymbol{\sigma}(\boldsymbol{x}, t) d V \\
& \boldsymbol{E}(t)=\frac{1}{V} \int_{V} \boldsymbol{\epsilon}(\boldsymbol{x}, t) d V \\
& \boldsymbol{\Omega}(t)=\frac{1}{V} \int_{V} \boldsymbol{\omega}(\boldsymbol{x}, t) d V
\end{aligned}
$$

The problem of finding the local fields $\boldsymbol{\sigma}(\boldsymbol{x}, t), \boldsymbol{\epsilon}(\boldsymbol{x}, t)$ and $\boldsymbol{\omega}(\boldsymbol{x}, t)$ consists of solving a particular set of differential equations resulting from compatibility and equilibrium conditions. More specifically, in the absence of volume forces, the respect of static equilibrium conditions imposes:

$\left.\operatorname{div}(\boldsymbol{\sigma})\right|_{\boldsymbol{x}, t}=\mathbf{0}$

Also, within the framework of infinitesimal strains and rotations, for compatibility conditions to be satisfied, the strain and rotation fields have to derive from the displacement field:

$\boldsymbol{\epsilon}(\boldsymbol{x}, t)=\operatorname{sym}\left(\left.\operatorname{grad}(\boldsymbol{u})\right|_{\boldsymbol{x}, t}\right)$

$\boldsymbol{\omega}(\boldsymbol{x}, t)=\operatorname{skw}\left(\left.\operatorname{grad}(\boldsymbol{u})\right|_{\boldsymbol{x}, t}\right)$

The resolution of the above differential equations requires specifying the constitutive relations associated with each material point. In the present work, we restrict ourselves to constitutive models for which the local strain and rotation tensors can be decomposed into elastic (superscript $e$ ) and inelastic ${ }^{1}$ (superscript in) contributions:

$\boldsymbol{\epsilon}(\boldsymbol{x}, t)=\boldsymbol{\epsilon}^{e}(\boldsymbol{x}, t)+\boldsymbol{\epsilon}^{i n}(\boldsymbol{x}, t)$

$\boldsymbol{\omega}(\boldsymbol{x}, t)=\boldsymbol{\omega}^{e}(\boldsymbol{x}, t)+\boldsymbol{\omega}^{i n}(\boldsymbol{x}, t)$

In the context of linear elasticity, the microscopic stress tensor $\boldsymbol{\sigma}(\boldsymbol{x}$, $t)$ is connected to the elastic strain tensor $\boldsymbol{\epsilon}^{e}(\boldsymbol{x}, t)$ according to:

$\boldsymbol{\sigma}(\boldsymbol{x}, t)=\mathbb{C}(\boldsymbol{x}, t): \boldsymbol{\epsilon}^{e}(\boldsymbol{x}, t)$

where $\mathbb{C}$ is the elastic stiffness tensor and $\mathbb{S}$ (with $\mathbb{S}=\mathbb{C}^{-1}$ ) is the elastic compliance tensor.

The introduction of a reference elastic homogeneous medium, with stiffness $\mathbb{C}^{0}$, allows the reformulation of the above problem in the form of a set of integral equations:

$\boldsymbol{\epsilon}(\boldsymbol{x}, t)=\boldsymbol{E}(t)-\int_{V} \mathbb{G}_{s y m}\left(\boldsymbol{x}-\boldsymbol{x}^{\prime}\right): \boldsymbol{\tau}\left(\boldsymbol{x}^{\prime}, t\right) d V$

$\boldsymbol{\omega}(\boldsymbol{x}, t)=\boldsymbol{\Omega}(t)-\int_{V} \mathbb{G}_{s k w}\left(\boldsymbol{x}-\boldsymbol{x}^{\prime}\right): \boldsymbol{\tau}\left(\boldsymbol{x}^{\prime}, t\right) d V$

where $\mathbb{G}_{s y m}$ (respectively $\mathbb{G}_{s k w}$ ) is the symmetric (respectively skew-symmetric) modified Green operator associated with the reference medium and $\boldsymbol{\tau}$ is the polarization field whose definition is:

$\boldsymbol{\tau}(\boldsymbol{x}, t)=\boldsymbol{\sigma}(\boldsymbol{x}, t)-\mathbb{C}^{0}: \boldsymbol{\epsilon}(\boldsymbol{x}, t)$

In the present work, the reference medium is assumed to be isotropic with a bulk modulus $k^{0}$ and a shear modulus $\mu^{0}$. These properties are chosen according to the suggestion of Eyre and Milton (1999):

$k^{0}=\sqrt{k_{\min } k_{\max }}$

$\mu^{0}=\sqrt{\mu_{\min } \mu_{\max }}$

where $k_{\min }$ and $k_{\max }$ (respectively $\mu_{\min }$ and $\mu_{\max }$ ) are the minimum and maximum values of the bulk modulus (respectively shear modulus) in $V$.

\footnotetext{
${ }^{1}$ No particular restriction applies to the origin of the inelastic contribution (e.g. thermal, plastic, viscoplastic).
} 


\section{FFT-based resolution method}

In most situations, no analytical solution is available for the integral Eqs. (11) and (12). To obtain a numerical solution, the volume element is discretized into $N$ voxels with volume $v$. For a given voxel, the corresponding barycentric position $\overline{\boldsymbol{x}}$ is:

$\overline{\boldsymbol{x}}=\frac{1}{v} \int_{v} \boldsymbol{x} d v$

The discrete form of Eqs. (11) and (12) is then given by:

$\overline{\boldsymbol{\epsilon}}(\overline{\boldsymbol{x}}, t)=\boldsymbol{E}(t)-\sum_{N} \mathbb{G}_{s y m}\left(\overline{\boldsymbol{x}}-\overline{\boldsymbol{x}}^{\prime}\right): \overline{\boldsymbol{\tau}}\left(\overline{\boldsymbol{x}}^{\prime}, t\right)$

$\overline{\boldsymbol{\omega}}(\overline{\boldsymbol{x}}, t)=\boldsymbol{\Omega}(t)-\sum_{N} \mathbb{G}_{s k w}\left(\overline{\boldsymbol{x}}-\overline{\boldsymbol{x}}^{\prime}\right): \overline{\boldsymbol{\tau}}\left(\overline{\boldsymbol{x}}^{\prime}, t\right)$

where $\overline{\boldsymbol{\epsilon}}(\overline{\boldsymbol{x}}, t), \overline{\boldsymbol{\omega}}(\overline{\boldsymbol{x}}, t), \quad \overline{\boldsymbol{\sigma}}(\overline{\boldsymbol{x}}, t)$ and $\overline{\boldsymbol{\tau}}(\overline{\boldsymbol{x}}, t)=\overline{\boldsymbol{\sigma}}(\overline{\boldsymbol{x}}, t)-\mathbb{C}^{0}: \overline{\boldsymbol{\epsilon}}(\overline{\boldsymbol{x}}, t)$ represent respectively the strain tensor, the rotation tensor, the stress tensor and the polarization tensor associated with a voxel of position $\overline{\boldsymbol{x}}$ at time $t$. A convenient way of evaluating the convolution operations involved in the above equations consists of using the discrete Fourier transform. Indeed, the application of the Fourier transform, which is denoted by a ' ', symbol, to Eqs. (17) and (18) leads to:

$\hat{\overline{\boldsymbol{\epsilon}}}(\boldsymbol{\xi}, t)= \begin{cases}\boldsymbol{E}(t) & \boldsymbol{\xi}=\mathbf{0} \\ -\hat{\mathbb{G}}_{\text {sym }}(\xi): \hat{\overline{\boldsymbol{\tau}}}(\boldsymbol{\xi}, t) & \boldsymbol{\xi} \neq \mathbf{0}\end{cases}$

$\hat{\overline{\boldsymbol{\omega}}}(\boldsymbol{\xi}, t)= \begin{cases}\boldsymbol{\Omega}(t) & \boldsymbol{\xi}=\mathbf{0} \\ -\hat{\mathbb{G}}_{s k w}(\boldsymbol{\xi}): \hat{\overline{\boldsymbol{\tau}}}(\boldsymbol{\xi}, t) & \boldsymbol{\xi} \neq \mathbf{0}\end{cases}$

where $\boldsymbol{\xi}$ denotes the frequency vector. The integral Eqs. (17) and (18) are thus solved in the frequency domain, and the application of the inverse Fourier transform leads to the expression of the fields $\overline{\boldsymbol{\epsilon}}(\overline{\boldsymbol{x}}, t)$ and $\overline{\boldsymbol{\omega}}(\overline{\boldsymbol{x}}, t)$ in the original spatial domain. However, because the polarization field $\overline{\boldsymbol{\tau}}(\overline{\boldsymbol{x}}, t)$ depends on the strain field $\overline{\boldsymbol{\epsilon}}(\overline{\boldsymbol{x}}, t)$, an iterative procedure is generally required to solve (19). In this work, the procedure proposed by Eyre and Milton (1999), which is detailed in Appendix A, is used to solve (19).

Also, for the resolution of Eqs. (19) and (20), the expressions of the modified Green operators in the frequency domain need to be known. In this work, a centered finite difference approximation is used to construct these operators from compatibility and equilibrium conditions (Willot, 2015).

\section{Composite voxel methods}

When discretizing a volume element, the application of the above FFT-based resolution method requires to define the properties associated with each voxel. While the common method consists of assigning the properties of the predominant constituent, more sophisticated strategies can be used. Here, to deal with twoscale microstructures, voxels are decomposed into different complementary domains corresponding to different constituents. Each sub-domain, say $\alpha$ (with $\alpha=1$ to $M$ ), has homogeneous properties. For each voxel of volume $v$, the introduction of a set of indicator functions $\eta^{\alpha}(\boldsymbol{x}, \overline{\boldsymbol{x}}, t)$ allows the calculation of the volume fractions $\phi^{\alpha}$ of the different constituents:

$\phi^{\alpha}(\overline{\boldsymbol{x}}, t)=\frac{1}{v} \int_{v} \eta^{\alpha}(\boldsymbol{x}, \overline{\boldsymbol{x}}, t) d v$

The indicator functions $\eta^{\alpha}(\boldsymbol{x}, \overline{\boldsymbol{x}}, t)$ take a unity value when the position $\boldsymbol{x}$ is included in the $\alpha$ th domain of the voxel with barycentric position $\overline{\boldsymbol{x}}$ and a zero value elsewhere. For the $\alpha$ th domain of the voxel with barycentric position $\overline{\boldsymbol{x}}$, the average stress, strain and rotation fields at time $t$ are denoted by $\boldsymbol{\sigma}^{\alpha}(\overline{\boldsymbol{x}}, t), \boldsymbol{\epsilon}^{\alpha}(\overline{\boldsymbol{x}}, t)$ and $\boldsymbol{\omega}^{\alpha}(\overline{\boldsymbol{x}}, t)$.
Assuming the characteristic length scales associated with the different constituents are inferior to the size of a voxel, the effective stress, strain and rotation tensors are then defined from the averaging relations:

$\overline{\boldsymbol{\sigma}}(\overline{\boldsymbol{x}}, t)=\sum_{\alpha}\left(\phi^{\alpha}(\overline{\boldsymbol{x}}, t) \boldsymbol{\sigma}^{\alpha}(\overline{\boldsymbol{x}}, t)\right)$

$\overline{\boldsymbol{\epsilon}}(\overline{\boldsymbol{x}}, t)=\sum_{\alpha}\left(\phi^{\alpha}(\overline{\boldsymbol{x}}, t) \boldsymbol{\epsilon}^{\alpha}(\overline{\boldsymbol{x}}, t)\right)$

$\overline{\boldsymbol{\omega}}(\overline{\boldsymbol{x}}, t)=\sum_{\alpha}\left(\phi^{\alpha}(\overline{\boldsymbol{x}}, t) \boldsymbol{\omega}^{\alpha}(\overline{\boldsymbol{x}}, t)\right)$

For each constituent, the stress tensor $\boldsymbol{\sigma}^{\alpha}(\overline{\boldsymbol{x}}, t)$ is connected to the strain tensor $\boldsymbol{\epsilon}^{\alpha}(\overline{\boldsymbol{x}}, t)$ according to an inelastic type of constitutive relation:

$\boldsymbol{\sigma}^{\alpha}(\overline{\boldsymbol{x}}, t)=\mathbb{C}^{\alpha}(\overline{\boldsymbol{x}}, t):\left(\boldsymbol{\epsilon}^{\alpha}(\overline{\boldsymbol{x}}, t)-\boldsymbol{\epsilon}^{i n, \alpha}(\overline{\boldsymbol{x}}, t)\right)$

where $\mathbb{C}^{\alpha}(\overline{\boldsymbol{x}}, t)$ and $\boldsymbol{\epsilon}^{i n, \alpha}(\overline{\boldsymbol{x}}, t)$ are the elastic stiffness tensor and the inelastic strain tensor for the $\alpha$ th constituent.

In the following, different homogenization methods for assigning properties to heterogeneous voxels are presented. For a given voxel, these methods aim at finding the effective behavior which is given by the effective elastic stiffness tensor $\overline{\mathbb{C}}(\overline{\boldsymbol{x}}, t)$ and the effective inelastic strain tensor $\overline{\boldsymbol{\epsilon}}^{i n}(\overline{\boldsymbol{x}}, t)$. These tensors connect the effective stress tensor to the effective strain tensor according to:

$\overline{\boldsymbol{\sigma}}(\overline{\boldsymbol{x}}, t)=\overline{\mathbb{C}}(\overline{\boldsymbol{x}}, t):\left(\overline{\boldsymbol{\epsilon}}(\overline{\boldsymbol{x}}, t)-\overline{\boldsymbol{\epsilon}}^{i n}(\overline{\boldsymbol{x}}, t)\right)$

When inelastic strains are treated as eigenstrains, the problem of finding $\overline{\mathbb{C}}(\overline{\boldsymbol{x}}, t)$ and $\overline{\boldsymbol{\epsilon}}^{i n}(\overline{\boldsymbol{x}}, t)$ is solved using the general equations for the homogenization of heterogeneous linear thermoelastic media. According to the classical result of homogenization theory of Rosen and Hashin (1970), and whatever the retained homogenization method is, the effective stiffness tensor $\overline{\mathbb{C}}(\overline{\boldsymbol{x}}, t)$ and the effective inelastic strain tensor $\overline{\boldsymbol{\epsilon}}^{i n}(\overline{\boldsymbol{x}}, t)$ are given by:

$\overline{\mathbb{C}}(\overline{\boldsymbol{x}}, t)=\sum_{\alpha} \phi^{\alpha}(\overline{\boldsymbol{x}}, t) \mathbb{C}^{\alpha}(\overline{\boldsymbol{x}}, t): \mathbb{A}^{\alpha}(\overline{\boldsymbol{x}}, t)$

$\overline{\boldsymbol{\epsilon}}^{i n}(\overline{\boldsymbol{x}}, t)=\sum_{\alpha} \phi^{\alpha}(\overline{\boldsymbol{x}}, t) \mathbb{B}^{\alpha^{T}}(\overline{\boldsymbol{x}}, t): \boldsymbol{\epsilon}^{i n, \alpha}(\overline{\boldsymbol{x}}, t)$

where $\mathbb{A}^{\alpha}$ and $\mathbb{B}^{\alpha}$ are the elastic strain and stress concentration tensors for the $\alpha$ th constituent. These tensors, which need to be specified for each homogenization method, are connected to each other with the following relation:

$\mathbb{B}^{\alpha}(\overline{\boldsymbol{x}}, t)=\mathbb{C}^{\alpha}(\overline{\boldsymbol{x}}, t): \mathbb{A}^{\alpha}(\overline{\boldsymbol{x}}, t): \overline{\mathbb{S}}(\overline{\boldsymbol{x}}, t)$

where $\overline{\mathbb{S}}=\overline{\mathbb{C}}^{-1}$ is the effective elastic compliance tensor.

In the following, for convenience, the dependence with position and time will be omitted unless needed.

\subsection{Voigt approximation}

For a given voxel, a simple approximation consists of considering the strain and rotation fields to be uniform within $v$. Thus, localization equations, which connect $\boldsymbol{\epsilon}^{\alpha}$ and $\boldsymbol{\omega}^{\alpha}$ to $\overline{\boldsymbol{\epsilon}}$ and $\overline{\boldsymbol{\omega}}$, are:

$\boldsymbol{\epsilon}^{\alpha}=\overline{\boldsymbol{\epsilon}}$

$\omega^{\alpha}=\bar{\omega}$

The elastic strain concentration tensor $\mathbb{A}^{\alpha}$, which is needed to compute the effective elastic stiffness tensor and the effective inelastic strain tensor, is given for each domain by:

$\mathbb{A}^{\alpha}=\mathbb{I}$ 
where $\mathbb{I}$ is the fourth rank identity tensor.

While the Voigt approximation is straightforward to implement, it has two major drawbacks. First, the influence of morphological factors is completely ignored, only the volume fractions are considered. Second, it is an upper bound approximation which often leads to an important overestimation of internal stresses.

\subsection{Laminate structure approximation}

Another approximation is obtained by treating a heterogeneous voxel as a laminate structure. For such a voxel, the planar interfaces separating the different constituents have the same normal direction, which is defined by the unitary vector $\boldsymbol{k}$. Considering the interfaces to be perfect, the continuity of the traction vector imposes:

$\boldsymbol{\sigma}^{\alpha} \cdot \boldsymbol{k}=\overline{\boldsymbol{\sigma}} \cdot \boldsymbol{k}$

Also, for the strain and rotation tensors to be compatible, the following equalities must hold:

$\boldsymbol{\epsilon}^{\alpha}:(\boldsymbol{l} \otimes \boldsymbol{l})=\overline{\boldsymbol{\epsilon}}:(\boldsymbol{l} \otimes \boldsymbol{l})$

$\boldsymbol{\epsilon}^{\alpha}:(\boldsymbol{m} \otimes \boldsymbol{m})=\overline{\boldsymbol{\epsilon}}:(\boldsymbol{m} \otimes \boldsymbol{m})$

$\left(\boldsymbol{\epsilon}^{\alpha}+\boldsymbol{\omega}^{\alpha}\right):(\boldsymbol{l} \otimes \boldsymbol{m})=(\overline{\boldsymbol{\epsilon}}+\overline{\boldsymbol{\omega}}):(\boldsymbol{l} \otimes \boldsymbol{m})$

$\left(\boldsymbol{\epsilon}^{\alpha}+\boldsymbol{\omega}^{\alpha}\right):(\boldsymbol{k} \otimes \boldsymbol{l})=(\overline{\boldsymbol{\epsilon}}+\overline{\boldsymbol{\omega}}):(\boldsymbol{k} \otimes \boldsymbol{l})$

$\left(\boldsymbol{\epsilon}^{\alpha}+\boldsymbol{\omega}^{\alpha}\right):(\boldsymbol{k} \otimes \boldsymbol{m})=(\overline{\boldsymbol{\epsilon}}+\overline{\boldsymbol{\omega}}):(\boldsymbol{k} \otimes \boldsymbol{m})$

In the above equations, $\boldsymbol{l}$ and $\boldsymbol{m}$ are two mutually orthogonal vectors which are orthogonal to $\boldsymbol{k}$. The complete set of strain and rotation localization equations is obtained by introducing constitutive relations into Eq. (33):

$\left(\mathbb{C}^{\alpha}:\left(\boldsymbol{\epsilon}^{\alpha}-\boldsymbol{\epsilon}^{i n, \alpha}\right)\right) \cdot \boldsymbol{k}=\left(\overline{\mathbb{C}}:\left(\overline{\boldsymbol{\epsilon}}-\overline{\boldsymbol{\epsilon}}^{i n}\right)\right) \cdot \boldsymbol{k}$

For a given voxel with prescribed strain and rotation tensors $(\overline{\boldsymbol{\epsilon}}$ and $\overline{\boldsymbol{\omega}})$, relations (34) to (39) allow the determination of the strain and rotation tensors associated with the $\alpha$ th constituent. The application of relation (39) however requires the knowledge of the effective elastic stiffness tensor $\overline{\mathbb{C}}$ and the effective inelastic strain tensor $\overline{\boldsymbol{\epsilon}}^{\text {in }}$. To compute these tensors, relations (34) to (39) are grouped according to:

$\mathbb{F}^{\alpha}: \boldsymbol{\epsilon}^{\alpha}-\mathbb{H}^{\alpha}: \boldsymbol{\epsilon}^{i n, \alpha}=\overline{\mathbb{F}}: \overline{\boldsymbol{\epsilon}}-\overline{\mathbb{H}}: \overline{\boldsymbol{\epsilon}}^{i n}$

The elastic strain concentration tensor $\mathbb{A}^{\alpha}$ for the $\alpha$ th domain is then deduced from the averaging condition (23):

$\mathbb{A}^{\alpha}=\mathbb{F}^{\alpha^{-1}}:\left(\sum_{\alpha}\left(\phi^{\alpha} \mathbb{F}^{\alpha^{-1}}\right)\right)^{-1}$

The effective stiffness tensor $\overline{\mathbb{C}}$ and the effective inelastic strain tensor $\overline{\boldsymbol{\epsilon}}^{i n}$ are finally obtained from relations (27) and (28). With the above definition of the elastic strain concentration tensors, the expression of the effective stiffness tensor $\overline{\mathbb{C}}$ is valid for any class of material symmetry; it is equivalent to the general expression proposed by Milton (2002) for laminate composite materials.

In contrast with the Voigt approximation, the laminate structure approximation considers some kind of morphological information to determine the effective behavior of a given voxel. Though this approach does not require any sophisticated numerical procedure, it is restricted to lamellar structures.

\subsection{Mori-Tanaka approximation}

For some voxels, a matrix-inclusion type of structure can sometimes be assumed. In such situations, if the matrix constituent represents a large portion of the heterogeneous voxel, the MoriTanaka approximation (Mori and Tanaka, 1973) can be used to estimate the corresponding effective properties. For the matrix constituent, the superscript $\alpha=\mu$ is used while inclusion constituents are denoted by the superscript $\alpha=\beta$. According to the MoriTanaka approximation, the strain and rotation tensors for inclusion constituents are given by:

$\boldsymbol{\epsilon}^{\beta}=\mathbb{A}^{\star \beta}: \boldsymbol{\epsilon}^{\mu}+\mathbb{A}^{\star \beta}: \mathbb{E}_{s y m}^{\beta}: \mathbb{S}^{\mu}:\left(\mathbb{C}^{\beta}: \boldsymbol{\epsilon}^{i n, \beta}-\mathbb{C}^{\mu}: \boldsymbol{\epsilon}^{i n, \mu}\right)$

$\boldsymbol{\omega}^{\beta}=\boldsymbol{\omega}^{\mu}+\mathbb{E}_{s k w}^{\beta}: \mathbb{E}_{s y m}^{\beta^{-1}}:\left(\boldsymbol{\epsilon}^{\beta}-\boldsymbol{\epsilon}^{\mu}\right)$

where $\mathbb{E}_{s y m}^{\beta}$ and $\mathbb{E}_{s k w}^{\beta}$ are the symmetric and skew-symmetric Eshelby tensors associated with the $\beta$ th inclusion. The tensor $\mathbb{A}^{\star \beta}$ is calculated from:

$\mathbb{A}^{\star \beta}=\left(\mathbb{I}+\mathbb{E}_{\text {sym }}^{\beta}: \mathbb{S}^{\mu}:\left(\mathbb{C}^{\beta}-\mathbb{C}^{\mu}\right)\right)^{-1}$

The strain and rotation tensors for the matrix constituent ( $\epsilon^{\mu}$ and $\left.\boldsymbol{\omega}^{\mu}\right)$ are obtained from the averaging conditions (23) and (24):

$\boldsymbol{\epsilon}^{\mu}=\mathbb{A}^{\star \mu}: \overline{\boldsymbol{\epsilon}}-\mathbb{A}^{\star \mu}: \sum_{\beta}\left(\phi^{\beta} \mathbb{A}^{\star \beta}: \mathbb{E}_{\text {sym }}^{\beta}: \mathbb{S}^{\mu}:\left(\mathbb{C}^{\beta}: \boldsymbol{\epsilon}^{i n, \beta}-\mathbb{C}^{\mu}: \boldsymbol{\epsilon}^{i n, \mu}\right)\right)$

$\boldsymbol{\omega}^{\mu}=\overline{\boldsymbol{\omega}}-\sum_{\beta}\left(\phi^{\beta} \mathbb{E}_{s k w}^{\beta}: \mathbb{E}_{s y m}^{\beta^{-1}}:\left(\boldsymbol{\epsilon}^{\beta}-\boldsymbol{\epsilon}^{\mu}\right)\right)$

with:

$\mathbb{A}^{\star \mu}=\left(\sum_{\beta}\left(\phi^{\beta} \mathbb{A}^{\star \beta}\right)+\phi^{\mu} \mathbb{I}\right)^{-1}$

The following expression of the elastic strain concentration tensor is used to determine the effective stiffness tensor $\overline{\mathbb{C}}$ and the effective inelastic strain tensor $\overline{\boldsymbol{\epsilon}}^{\text {in }}$ :

$\mathbb{A}^{\alpha}= \begin{cases}\mathbb{A}^{\star \mu}, & \alpha=\mu \\ \mathbb{A}^{\star \beta}: \mathbb{A}^{\star \mu}, & \alpha=\beta\end{cases}$

In some specific situations, the Mori-Tanaka approximation provides an accurate estimation of the effective properties associated with a heterogeneous voxel. Nevertheless, the Eshelby tensor $\mathbb{E}_{\text {sym }}^{\alpha}$ has to be known to compute the effective properties. While some analytical expressions are available for ellipsoidal inclusions when the elastic properties of the matrix material are isotropic (Mura, 1987), a numerical procedure is needed to evaluate these tensors in the general case.

\section{Application to pearlitic microstructures}

In this section, the mechanical behavior of a fully pearlitic eutectoid steel is investigated. Pearlite is a lamellar aggregate with alternating layers of cementite and ferrite for which the characteristic length scale is typically about a few hundreds of nanometers (Marder and Bramfitt, 1976; Dollar et al., 1988; Yahyaoui et al., 2014), much lower than the typical grain size of pearlitic grains. In equilibrium conditions, the volume fractions of ferrite and cementite are respectively about $88 \%$ and $12 \%$. Since the explicit discretization of pearlitic microstructures is often not possible, composite voxel methods provide a solution for dealing with such fine microstructures. 


\subsection{Constitutive model}

For a given constituent (i.e. ferrite or cementite), a ratedependent elasto-plastic behavior is assumed. Adopting the general framework of crystal plasticity for rate-independent processes (Hill, 1966), a set of $S$ slip systems is introduced to express the inelastic contribution. Each slip system $s$ (with $s=1$ to $S$ ) is defined with a slip direction $\boldsymbol{b}^{s, \alpha}$ and a slip plane normal $\boldsymbol{n}^{s, \alpha}$. The inelastic strain and rotation tensors $\left(\epsilon^{p, \alpha}\right.$ and $\left.\omega^{p, \alpha}\right)$ are expressed as a function of the plastic shear strains $\gamma^{s, \alpha}$ according to:

$\boldsymbol{\epsilon}^{i n, \alpha}=\sum_{s} \operatorname{sym}\left(\boldsymbol{b}^{s, \alpha} \otimes \boldsymbol{n}^{s, \alpha}\right) \gamma^{s, \alpha}$

$\boldsymbol{\omega}^{i n, \alpha}=\sum_{s} \operatorname{skw}\left(\boldsymbol{b}^{s, \alpha} \otimes \boldsymbol{n}^{s, \alpha}\right) \gamma^{s, \alpha}$

The shear stress $\tau^{s, \alpha}$ acting on a given slip system is obtained from the projection of the stress tensor $\sigma^{\alpha}$ :

$\tau^{s, \alpha}=\boldsymbol{b}^{s, \alpha} \cdot \boldsymbol{\sigma}^{\alpha} \cdot \boldsymbol{n}^{s, \alpha}$

For each slip system, plastic flow is possible only when the critical shear stress $g^{s, \alpha}$ is reached. The evolution of the critical shear stress is assumed to be linear:

$g^{s, \alpha}=g_{0}^{\alpha}+\sum_{q}\left(H^{s q, \alpha} p^{q, \alpha}\right)$

where $g_{0}^{\alpha}$ is the initial value of the critical shear stress, $H^{\alpha}$ is the hardening matrix and $p^{s, \alpha}$ is the accumulated plastic shear strain (i.e. $\dot{p}^{s, \alpha}=\left|\dot{\gamma}^{s, \alpha}\right|$ ). Within a rate-independent plasticity framework, active slip systems are defined according to the following conditions:

$\dot{\gamma}^{s, \alpha} \neq 0$,

$\left|\tau^{s, \alpha}\right|=g^{s, \alpha}$ and $\left|\dot{\tau}^{s, \alpha}\right|=\dot{g}^{s, \alpha}$

$\dot{\gamma}^{s, \alpha}=0$,

$$
\left|\tau^{s, \alpha}\right|<g^{s, \alpha} \text { or }\left|\dot{\tau}^{s, \alpha}\right|<\dot{g}^{s, \alpha}
$$

At each time $t$, for active slip systems, the corresponding plastic shear strain rates $\dot{\gamma}^{s, \alpha}$ are determined to fulfill the consistency condition $\left|\dot{\tau}^{s, \alpha}\right|=\dot{g}^{s, \alpha}$.

According to the above constitutive relations, the set of material parameters for a given constituent consists of the independent thermoelastic constants associated with $\mathbb{C}^{\alpha}$ and $\boldsymbol{\alpha}^{\alpha}$, the initial critical shear stress $g_{0}^{\alpha}$ and the hardening matrix $H^{\alpha}$.

\subsection{Representative volume element}

To represent the pearlitic steel aggregate, the volume element presented in Fig. 1 is used. It has been constructed from a Voronoï tessellation and consists of 200 equiaxed grains with random crystallographic orientations. For the application of the FFT-based resolution method, the volume element is discretized into $128^{3}$ voxels.

To describe pearlitic microstructures, three different assumptions are used: the Voigt approximation, the laminate structure approximation and the Mori-Tanaka approximation. For the application of the Mori-Tanaka approximation, ferrite is considered to be the matrix constituent and cementite lamellas are treated as penny-shaped inclusions with an aspect ratio of 20 .

The numerical procedures used for computing the effective behavior of a volume element with prescribed boundary conditions are detailed in Appendix A.

\subsection{Material parameters}

The elastic constants associated with cementite, for which a purely elastic behavior was assumed, were extracted from the work of Henriksson et al. (2008). For ferrite, both the $\{110\}\langle 111\rangle$

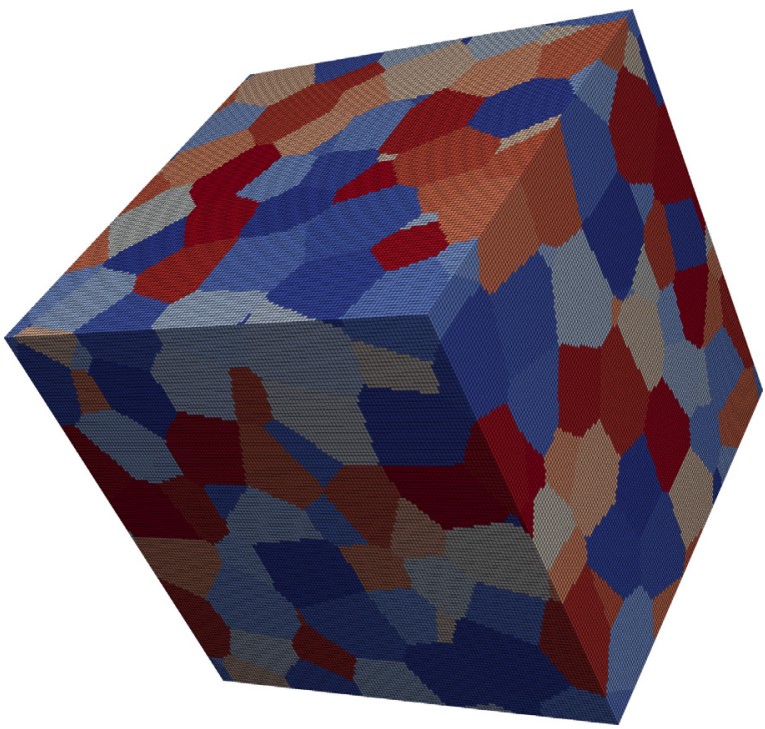

Fig. 1. Periodic representative volume element used for the different calculations. The volume element is discretized into $128^{3}$ voxels.

Table 1

Material parameters for the eutectoid steel grade. Only independent elastic constants are listed.

\begin{tabular}{lll}
\hline Cementite & & \\
\hline $\mathbb{C}_{11}(\mathrm{GPa})$ & $\mathbb{C}_{12}(\mathrm{GPa})$ & $\mathbb{C}_{13}(\mathrm{GPa})$ \\
394 & 157 & 146 \\
$\mathbb{C}_{22}(\mathrm{GPa})$ & $\mathbb{C}_{23}(\mathrm{GPa})$ & $\mathbb{C}_{33}(\mathrm{GPa})$ \\
412 & 166 & 360 \\
$\mathbb{C}_{44}(\mathrm{GPa})$ & $\mathbb{C}_{55}(\mathrm{GPa})$ & $\mathbb{C}_{66}(\mathrm{GPa})$ \\
83 & 133 & 136 \\
& Ferrite & \\
$\mathbb{C}_{11}(\mathrm{GPa})$ & $\mathbb{C}_{12}(\mathrm{GPa})$ & $\mathbb{C}_{44}(\mathrm{GPa})$ \\
231 & 135 & 116 \\
$g_{0}(\mathrm{MPa})$ & $H^{s s}(\mathrm{MPa})$ & $H^{s t}(\mathrm{MPa})$ \\
270 & 2.2 & 2.0 \\
\hline
\end{tabular}

and $\{112\}\langle 111\rangle$ slip systems have been considered. The corresponding hardening parameters have been chosen to match the experimental results of Inal et al. (2004) who used diffraction techniques to determine the evolution of the average axial stress in each phase during an uniaxial tension test. The material parameters associated with both phases are listed in Table 1.

\subsection{Results}

First, the effective response of the pearlitic polycrystalline aggregate has been computed for the case of uniaxial tension (up to an axial strain $E_{11}$ of $2 \%$ ). The evolution of the macroscopic axial stress $\Sigma_{11}$ as a function of the macroscopic axial strain $E_{11}$ is plotted in Fig. 2. The evolution of the average stress is given for each metallurgical phase (ferrite and cementite) in Figs. 3 and 4. According to the results, the effective hardening rate in the plastic regime is much higher when the Voigt approximation is used. This difference between the Voigt approximation and the other approximations can be attributed to internal stresses, which are largely overestimated with the Voigt approximation. Indeed, while the behavior of the ferritic phase is correctly described with all methods, important discrepancies exist concerning cementite. More specifically, the bilinear type of behavior which is experimentally ob- 


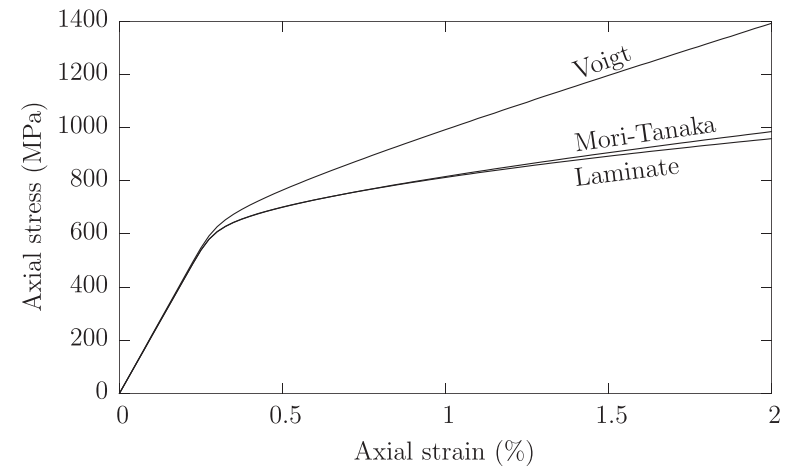

Fig. 2. Evolution of the macroscopic axial stress $\Sigma_{11}$ as a function of the macroscopic axial strain $E_{11}$ for a pearlitic steel polycrystalline aggregate subjected to uniaxial tension.

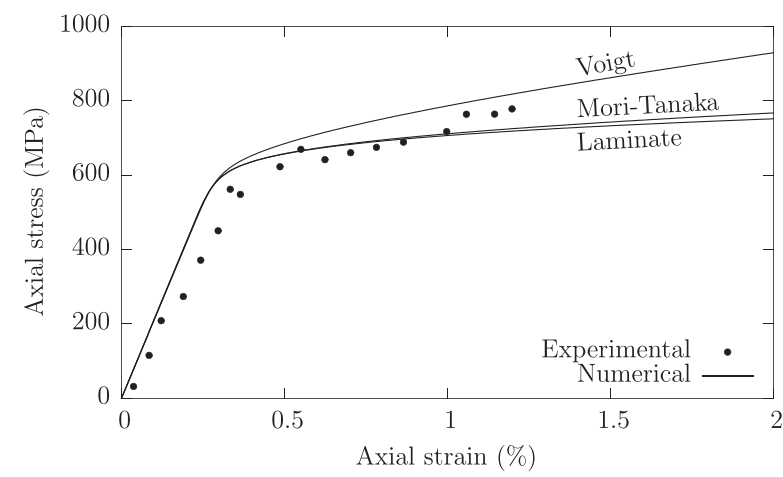

Fig. 3. Evolution of the average stress in ferrite as a function of the macroscopic axial strain $E_{11}$ for a pearlitic steel polycrystalline aggregate subjected to uniaxial tension. The experimental results were obtained by (Inal et al., 2004).

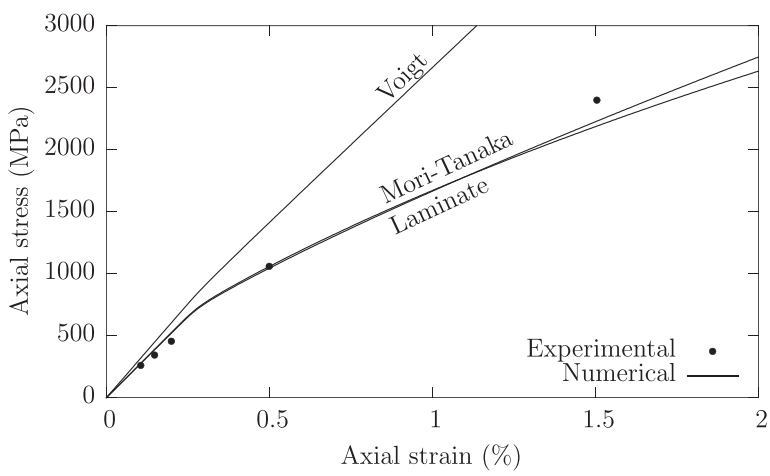

Fig. 4. Evolution of the average stress in cementite as a function of the macroscopic axial strain $E_{11}$ for a pearlitic steel polycrystalline aggregate subjected to uniaxial tension. The experimental results were obtained by (Inal et al., 2004).

served for cementite is reasonably reproduced only when the laminate structure or the Mori-Tanaka approximations are used. For a given voxel, the Voigt approximation imposes the same strain state in both constituents. It thus fails in describing the fact that strains are preferably transferred to the soft constituent (ferrite) rather than the hard constituent (cementite). As a consequence, important internal stresses are generated in cementite because the imposed strain state is accommodated in a purely elastic manner. These internal stresses provide a supplementary contribution to hardening, resulting in an overestimation of the final axial stress in cementite. These results thus emphasize the importance of a
Table 2

Total computation times required for performing uniaxial tension simulations for each method.

\begin{tabular}{ll}
\hline Method & Computation time $(\mathrm{s})$ \\
\hline Voigt & $1.27 \times 10^{5} \mathrm{~s}$ \\
Laminate & $2.16 \times 10^{5} \mathrm{~s}$ \\
Mori-Tanaka & $2.67 \times 10^{5} \mathrm{~s}$ \\
\hline
\end{tabular}

proper description of morphological features when evaluating the response of heterogeneous materials with multiple length scales.

The above computations have been performed on a DELL PowerEdge R710 server with 2 processors (Intel Xeon X5690) using 8 cores. The computation time required for performing uniaxial tension simulations is given for each method in Table 2. The lowest computation time is obtained with the Voigt approximation, which does not involve any sophisticated numerical procedure. At the opposite, important computational efforts are needed for the application of the Mori-Tanaka approximation. Indeed, for this homogenization method, the determination of effective properties requires additional operations with a significant impact on the total computation time.

Finally, the response of the pearlitic polycrystalline aggregate has been calculated for plane strain loading conditions such that the imposed macroscopic strain state is:

$\boldsymbol{E}=\left|\begin{array}{ccc}+10 \% & 0 & 0 \\ 0 & 0 & 0 \\ 0 & 0 & -10 \%\end{array}\right|$

The loading conditions are chosen to represent a rolling operation with a reduction ratio of $10 \%$. To show how rotations are impacted by the chosen homogenization method, the final $\{110\}$ and $\{111\}$ pole figures obtained for the ferritic phase after rolling are plotted in Fig. 5. Though quite similar trends are obtained for all approximations, texture evolution is found to be slightly more pronounced when the Voigt approximation is used.

\section{Conclusions}

When using FFT-based techniques for modelling the behavior of heterogeneous materials with periodic microstructures, the common strategy consists of assuming the mechanical properties to be homogeneous within each voxel. This assumption is however not sufficient for microstructures with multiple length scales for which some specific strategies have to be developed. In the first part of this paper, different methods for considering multiple microstructural length scales have been proposed for materials with an inelastic behavior. More specifically, three different strategies, which use elementary homogenization rules to determine the effective behavior of heterogeneous voxels, have been described: the Voigt approximation, the laminate structure approximation and the Mori-Tanaka approximation.

Then, to demonstrate their interest, these methods have been used to calculate the effective behavior of a pearlitic steel polycrystalline aggregate. According to the numerical results, a specific care should be taken when using the Voigt approximation. Indeed, the Voigt approximation, which is an upper bound approximation, strongly overestimates macroscopic stresses and ignores the influence of morphological features. The impact of such features on the effective behavior can be considered by using either the laminate structure approximation or the Mori-Tanaka approximation. The application of the Mori-Tanaka approximation however requires more important computational efforts. 


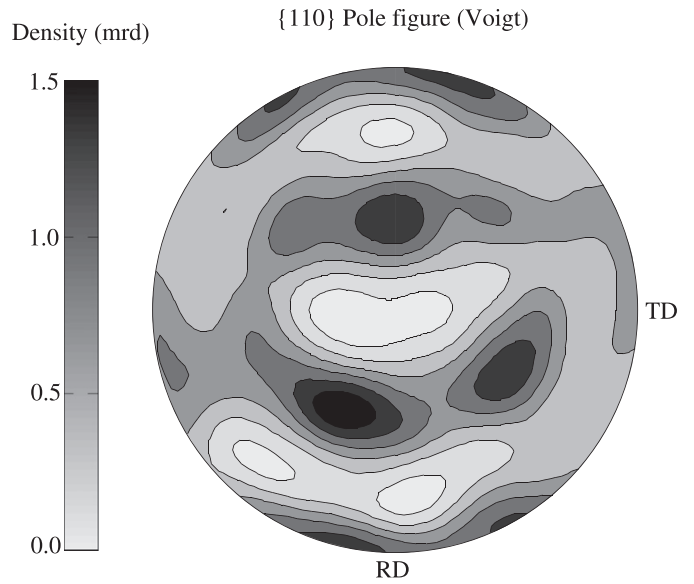

$\{110\}$ Pole figure (Laminate)

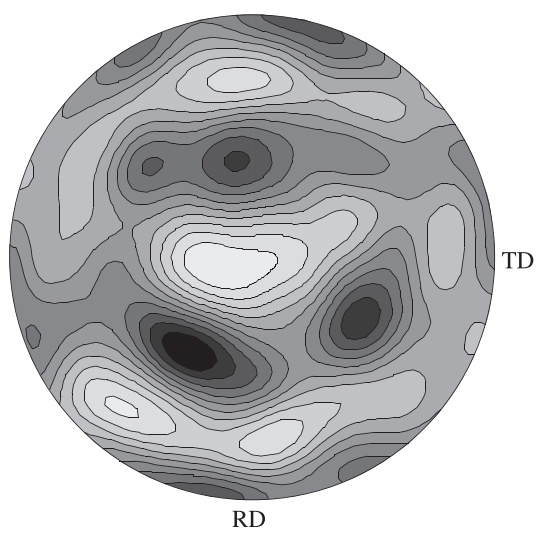

$\{110\}$ Pole figure (Mori-Tanaka)

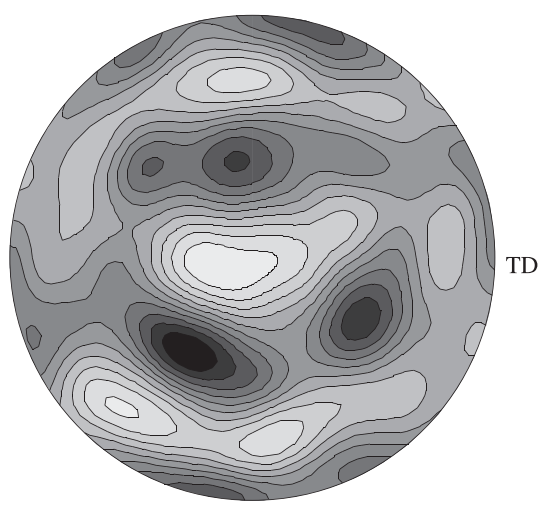

$\mathrm{RD}$
\{111\} Pole figure (Voigt)

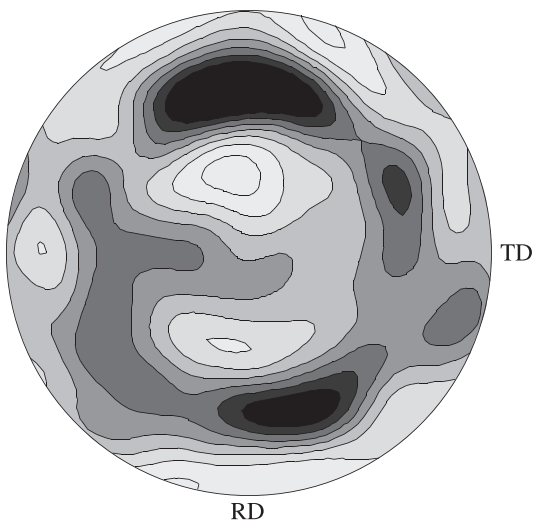

$\{111\}$ Pole figure (Laminate)

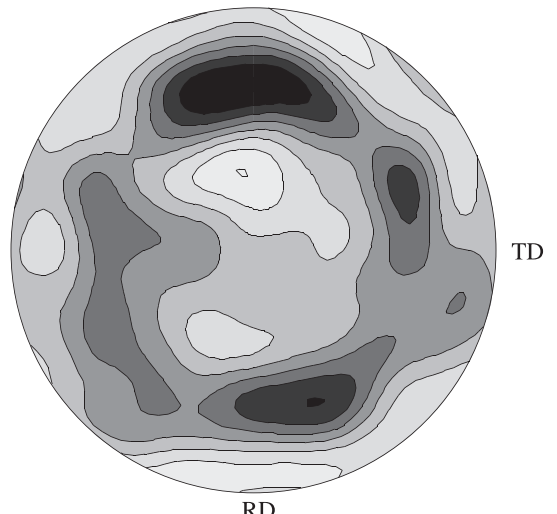

\{111\} Pole figure (Mori-Tanaka)

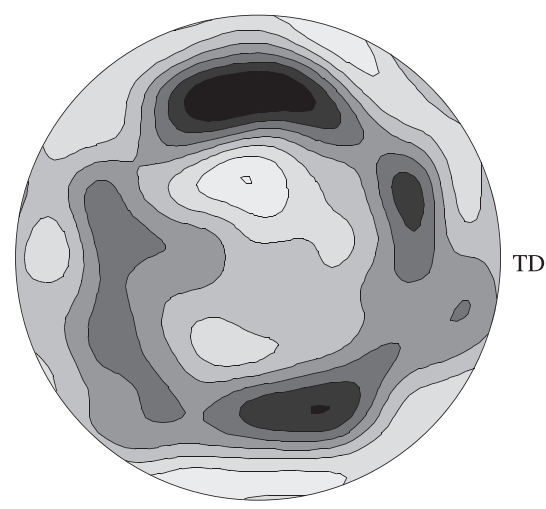

$\mathrm{RD}$

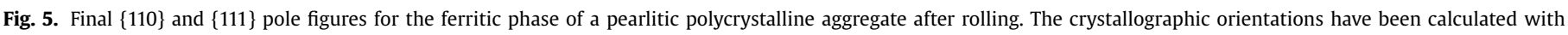
different approximations (Voigt, laminate structure or Mori-Tanaka). Pole figures have been plotted using the MTEX package (Hielscher and Schaeben, 2008).

\section{Appendix A. Implementation details}

The global strategy used for computing the effective behavior of a volume element with prescribed boundary conditions is described in Table A.3. The objective of the iterative procedure (from step 3a to step $3 \mathrm{~h}$ ) is twofold. First, it is needed to ensure that, once convergence is reached, the effective properties $\overline{\mathbb{C}}$ and $\overline{\boldsymbol{\epsilon}}^{\text {in }}$ used for steps $3 \mathrm{c}$ and $\mathrm{d}$ are the same as those determined from step 3f. Second, there are some situations where stress-controlled or mixed loading conditions are imposed to the volume element. However, whatever the boundary conditions are, step $3 c$ uses the macroscopic strain and rotation tensors $\boldsymbol{E}$ and $\boldsymbol{\Omega}$ to solve the integral Eqs. (11) and (12). The iterative procedure thus also aims at finding the macroscopic strain tensor $\boldsymbol{E}$ satisfying the imposed stress-controlled or mixed loading conditions.

Convergence is reached once the difference between the strain tensors obtained from two consecutive iterations is small enough. In the present work, a tolerance $e_{c}$ of $10^{-8}$ has been used for determining whether convergence is reached or not.

Also, one should note that the iterative procedure, which is needed for the resolution of the integral Eqs. (11) and (12), is contained in step 3c. In the present work, the resolution method pro- 
Table A.3

Description of the strategy used for computing the effective behavior of a volume element with FFT-based methods.

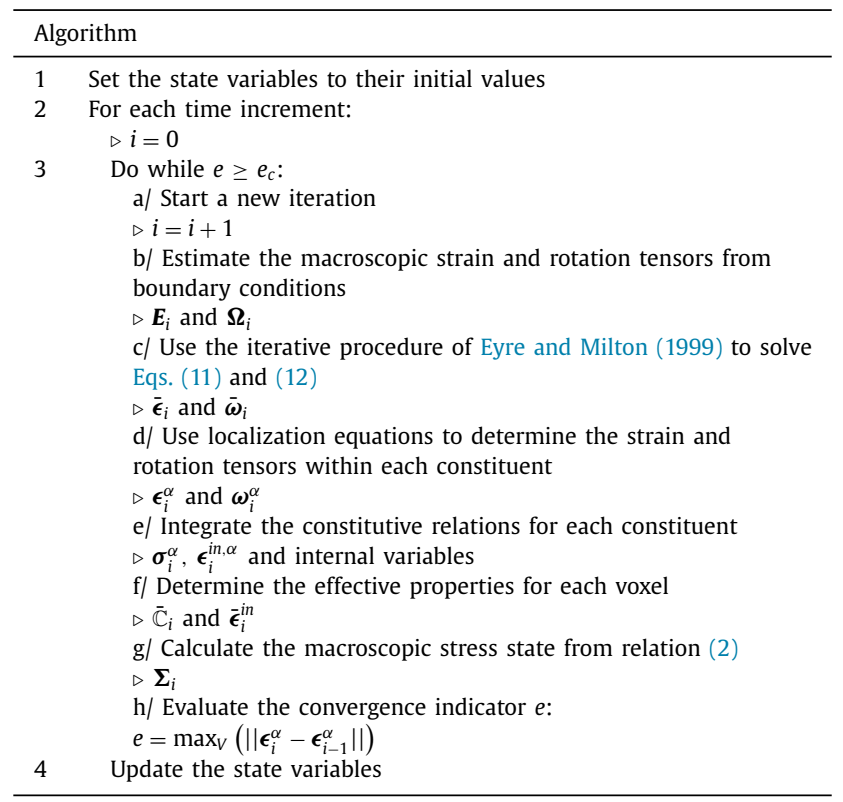

\section{Table A.4}

Description of the iterative procedure used for solving the integral Eqs. (11) and (12) with FFT-based methods.

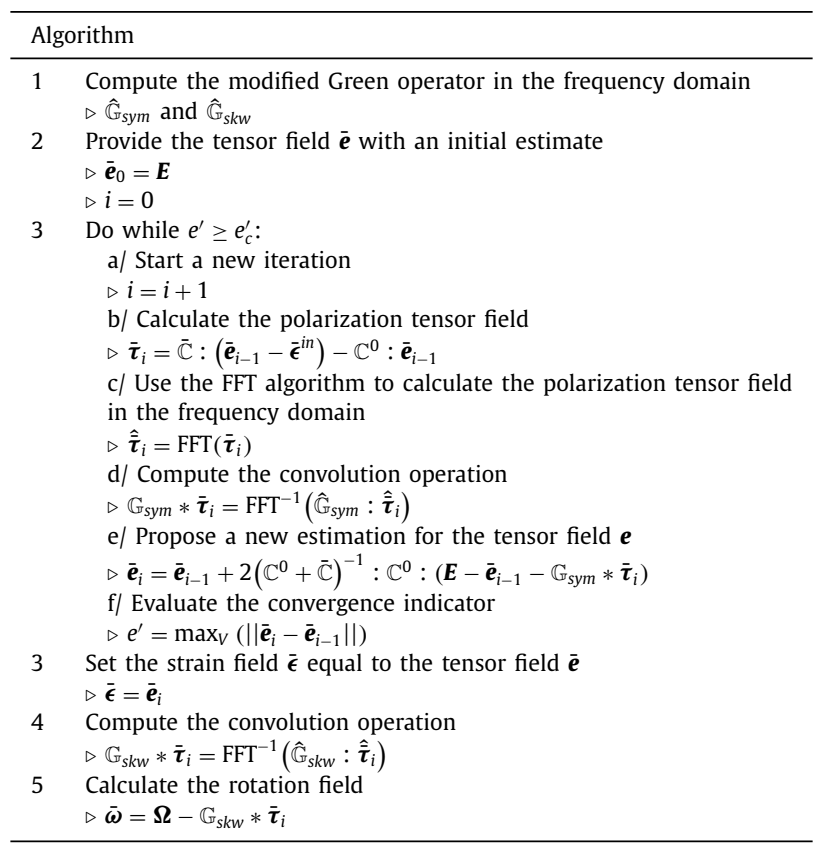

posed by Eyre and Milton (1999), which has been later reformulated by Michel et al. (2001), is used. A detailed description of the procedure is given in Table A.4. For the calculations presented in this work, a value of $10^{-9}$ is used for the convergence criterion $e_{c}^{\prime}$.

\section{References}

Anglin, B.S., Lebensohn, R.A., Rollett, A.D., 2014. Validation of a numerical method based on fast Fourier transforms for heterogeneous thermoelastic materials by comparison with analytical solutions. Comput. Mater. Sci 87, 209-217.
Berbenni, S., Taupin, V., Djaka, K.S., Fressengeas, C., 2014. A numerical spectral approach for solving elasto-static field dislocation and g-disclination mechanics, International Journal of Solids and Structures 51, 4157-4175.

Brenner, R., Beaudoin, A.J., Suquet, P., Acharya, A., 2014. Numerical implementation of static field dislocation mechanics theory for periodic media. Philos. Mag. 94, 1764-1787.

Brisard, S., Dormieux, L., 2012. Combining Galerkin approximation techniques with the principle of Hashin and Shtrikman to derive a new FFT-based numerica method for the homogenization of composites. Comput. Methods Appl. Mech. Eng. 217-220, 197-212.

Dollar, M., Bernstein, M., Thompson, A.W., 1988. Influence of deformation substructure on flow and fracture of fully pearlitic steel. Acta Metall 36. 311-20

Eisenlohr, P., Diehl, M., Lebensohn, R.A., Roters, F., 2013. A spectral method solution to crystal elasto-viscoplasticity at finite strains. Int. J. Plast. 46, 37-53.

Eyre, D.J., Milton, G.W., 1999. A fast numerical scheme for computing the response of composites using grid refinement. J. Phys. III 6, 41-47.

Figliuzzi, B., Jeulin, D., Faessel, M., Willot, F, Koishi, M., Kowatari, N., 2016. Modelling the microstructure and the viscoelastic behaviour of carbon black filled rubber materials from 3d simulations. Tech. Mecha. 36, 32-56.

Gélébart, L., Mondon-Cancel, R., 2013. Non-linear extension of FFT-based methods accelerated by conjugate gradients to evaluate the mechanical behavior of composite materials. Comput. Mater. Sci 77, 430-439.

Gélébart, L., Ouaki, F., 2015. Filtering material properties to improve FFT-based methods for numerical homogenization. J. Comput. Phys. 294, 90-95.

Henriksson, K.O.E., Sandberg, N., Wallenius, J., 2008. Carbides in stainless steels: results from ab initio investigations. Appl. Phys. Lett. 93, 191912.

Hielscher, R., Schaeben, H., 2008. A novel pole figure inversion method: specification of the MTEX algorithm. J. Appl. Cryst. 41, 1024-1037.

Hill, R., 1966. Generalized constitutive relations for incremental deformation of metal crystals by multislip. J. Mech. Phys. Solids 14, 95-102.

Inal, K., Lebrun, J.L., Belassel, M., 2004. Second-order stresses and strains in heterogeneous steels: self-consistent modeling and X-ray diffraction analysis. Metall. Mater. Trans. A 35A, 2361.

Kabel, M., Böhlke, T., Schneider, M., 2014. Efficient fixed point and Newton-Krylov solvers for FFT-based homogenization of elasticity at large deformations. Comput. Mech. 1-18.

Kabel, M., Fink, A., Ospald, F., Schneider, M., 2016a. Nonlinear Composite Voxels and FFT-Based Homogenization. In: Papadrakakis, M., Papadopoulos, V., Stefanou, G., Plevris, V. (Eds.), VII European Congress on Computational Methods in Applied Sciences and Engineering. Crete Island, Greece.

Kabel, M., Merkert, D., Schneider, M., 2015. Use of composite voxels in FFT-based homogenization. Comput. Methods Appl. Mech. Eng. 294, 168-188.

Kabel, M., Ospald, F., Schneider, M., 2016. A model order reduction method for computational homogenization at finite strains on regular grids using hyperelastic laminates to approximate interfaces. Comput. Methods Appl. Mech. Eng. 309, 476-496.

Lahellec, N., Michel, J.C., Moulinec, H., Suquet, P., 2003. Analysis of Inhomogeneous Materials at Large Strains Using Fast Fourier Transforms. In: Miehe, C. (Ed.), IUTAM Symposium on Computational Mechanics of Solid Materials at Large Strains. Springer Netherlands, Dordrecht, pp. 247-258.

Lebensohn, R.A., 2001. N-site modeling of a 3d viscoplastic polycrystal using fast Fourier transform. Acta Mater. 49, 2723-2737.

Lebensohn, R.A., Kanjarla, A.K., Eisenlohr, P., 2012. An elasto-viscoplastic formulation based on fast Fourier transforms for the prediction of micromechanical fields in polycrystalline materials. Int. J. Plast. 32-33, 59-69.

Lebensohn, R.A., Needleman, A., 2016. Numerical implementation of non-local polycrystal plasticity using fast Fourier transforms. J. Mech. Phys. Solids. In Press.

Marder, A.R., Bramfitt, B.L., 1976. The effect of morphology on the strength of pearlite. Metall. Trans. A 7A. 365-72.

Mareau, C., Cuillerer, D., Morel, F., 2013. Experimental and numerical study of the evolution of stored and dissipated energies in a medium carbon steel under cyclic loading. Mech. Mater. 60, 93-106.

Mareau, C., Daymond, M.R., 2016. Micromechanical modelling of twinning in polycrystalline materials: application to magnesium. Int. J. Plast. 85, 156-171.

Michel, J.C., Moulinec, H., Suquet, P., 2001. A computational scheme for linear and non-linear composites with arbitrary phase contrast. Int. J. Numer. Methods Eng 52, 139-160.

Milton, G.W., 2002. The theory of composites. Cambridge University Press. Cambridge Books Online.

Monchiet, V., Bonnet, G., 2012. A polarization-based FFT iterative scheme for computing the effective properties of elastic composites with arbitrary contrast. Int. J. Numer. Methods Eng. 89, 1419-1436.

Mori, T., Tanaka, K., 1973. Average stress in matrix and average elastic energy of materials with misfitting inclusions. Acta Metall. 21, 571-574.

Moulinec, H., Silva, F., 2014. Comparison of three accelerated FFT-based schemes for computing the mechanical response of composite materials. Int. J. Numer Methods Eng. 97, 960-985.

Moulinec, H., Suquet, P., 1998. A numerical method for computing the overall response of nonlinear composites with complex microstructure. Comput. Methods Appl. Mech. Eng. 157, 69-94.

Müller, W.H., 1996. Mathematical versus experimental stress analysis of inhomogeneities in solids. J. Phys. IV 6, 139-148.

Mura, T., 1987. Micromechanics of defects in solids. Kluwer Academic Publishers, Dordrecht, The Netherlands. 
Robert, C., Mareau, C., 2015. A comparison between different numerical methods for the modeling of polycrystalline materials with an elasticviscoplastic behavior. Comput. Mater. Sci 103, 134-144.

Rosen, B.W., Hashin, Z., 1970. Effective thermal expansion coefficients and specific heats of composite materials. Int. J. Eng. Sci. 8, 157-173.

Suquet, P., Moulinec, H., Castelnau, O., Montagnat, M., Lahellec, N., Grennerat, F., Duval, P., Brenner, R., 2012. Multi-scale modeling of the mechanical behavior of polycrystalline ice under transient creep. Procedia IUTAM 3, 76-90.

Vinogradov, V., Milton, G.W., 2008. An accelerated FFT algorithm for thermoelastic and non-linear composites. Int. J. Numer. Methods Eng. 76, 16781695.
Willot, F., 2015. Fourier-based schemes for computing the mechanical response of composites with accurate local fields. C. R. Mecanique 343, 232-245.

Willot, F., Pellegrini, Y.-P., 2008. Fast Fourier Transform Computations and Build-up of Plastic Deformation in 2D, Elastic-perfectly Plastic, Pixelwise Disordered Porous Media. In: Jeulin, D., Forest, S. (Eds.), Continuum Models and Discrete Systems CMDS 11. Ecole des Mines, Paris, pp. 443-449.

Yahyaoui, H., Sidhom, H., Braham, C., Baczmanski, A., 2014. Effect of interlamellar spacing on the elastoplastic behavior of $c 70$ pearlitic steel: experimental results and self-consistent modeling. Mater. Des. 55, 888-897.

Zeman, J., ejc, J.V., Novák, J., Marek, I., 2010. Accelerating a FFT-based solver for numerical homogenization of periodic media by conjugate gradients. J. Comput. Phys. 229, 8065-8071. 